\title{
Imaging the Subsurface with 3D inversion of Potential Field Data
}

\author{
Jun Wang ${ }^{1,2, \mathrm{a}^{*}}$, Xiaohong Meng ${ }^{1,2}$ and Fang $\mathrm{Li}^{1,2}$ \\ ${ }^{1}$ Key Laboratory of Geo-detection (China University of Geosciences, Beijing), Ministry of Education, \\ Beijing 100083, China \\ ${ }^{2}$ School of Geophysics and Information Technology, China University of Geosciences, Beijing \\ 100083, China \\ awangjun5505@163.com
}

Keywords: Imaging; Subsurface; 3D; Inversion; Potential Field Data.

\begin{abstract}
With the continuing growth in influence of near surface geophysics, the research of the surface geophysics is of great significance. Researchers want to understand the complex subsurface with gravity and magnetic data and inversion is a powerful tool. In this paper, researchers introduced how to formulate the 3D inversion of potential field data. Meanwhile, some other constrains such as depth-weighting are also employed to get more enhanced results. The method is applied to synthetic data for typical models of gravity anomalies.
\end{abstract}

\section{Introduction}

The continuing growth in resources demand has brought this area of geophysical detecting to the forefront. With geophysical sensing, subsurface structure and conditions are derived. Subsurface features vary with the geologic environments and getting a detailed understanding of it have great significance. With detail knowledge, researchers can find useful resources (oil, gas, metal mine et al.) and study underground structures to prevent geological disasters et al. How to get an accurate characterization of the subsurface is the main task of geophysicist.

With the observed data, how to know about the subsurface? Among the recently developed methodologies for data interpretation, it is geophysical inversion that works.

For generalized inversion methods of potential field data, the 3D earth is discretized into cells of constant density, susceptibility or a variant of the magnetization vector. At this point, finite number of mathematical equations is formulated to recover distribution of physical property parameters in the subspace. Physical parameters of these cells sketch out geological bodies as well as geological structures [1-3]. Over the years, a variety of methods have been developed for 3D inversion of potential field data with both smooth and focusing regularizations [4-8].

In this paper, researchers introduced how to formulate the 3D inversion of potential field data. Meanwhile, some other constrains such as depth-weighting are also employed to get more enhanced results. The method is applied to synthetic data for typical models of gravity anomalies.

\section{Formulation of the 3D inversion}

Just as mentioned above, density and magnetic susceptibility is uniform within each cell, the gravity and magnetic anomaly at a location on the surface is related to the subsurface density or susceptibility by a linear relationship. Here, researchers write them as

$$
\begin{aligned}
& d_{g}=G_{g} m \\
& d_{m}=G_{m} \kappa
\end{aligned}
$$

Where, $d_{g}$ is the observed gravity data vector, $d_{m}$ is the magnetic data vector, $m$ is density within each cell, and $\kappa$ is the susceptibility in each cell. Matrix $G_{g}$ and $G_{m}$ quantify the contribution of a fixed cell 
to a settled observation point.

The inverse problem is formulated as an optimization problem where an objective function of the model is minimized subject to some constraints, including Equation (1). Here researchers adopt a right-handed Cartesian coordinate system with $\mathrm{x}$ positive north and $\mathrm{z}$ positive down. Separate inversion takes the form as $\mathrm{Li}$ and Oldenburg $[4,5]$. That is the inverse problem formulated as follows: find a model $m$ that minimizes the model objective function subject to some constraints. Let the model objective function be

$$
\begin{aligned}
\phi(m)= & \alpha_{s} \int w_{s}\left\{w(z)\left[m-m_{r e f}\right]\right\}^{2} d v+\alpha_{x} \int w_{x}\left\{\frac{\partial w(z)}{\partial x}\left[m-m_{r e f}\right]\right\}^{2} d v+\ldots \\
& +\alpha_{z} \int w_{z}\left\{\frac{\partial w(z)}{\partial z}\left[m-m_{r e f}\right]\right\}^{2} d v \\
= & \alpha_{s}\left\|W_{s}\left(m-m_{r e f}\right)\right\|^{2}+\alpha_{x}\left\|W_{x}\left(m-m_{r e f}\right)\right\|^{2}+\alpha_{z}\left\|W_{z}\left(m-m_{r e f}\right)\right\|^{2} \\
= & \left\|W_{m}\left(m-m_{r e f}\right)\right\|^{2}
\end{aligned}
$$

Where, $\mathrm{w}_{s}, \mathrm{w}_{x}$ and $\mathrm{w}_{z}$ are spatially dependent weighting functions, and $W_{m}$ is the combined weighting matrix given by

$$
W_{m}^{T} W_{m}=\alpha_{s} W_{s}^{T} W_{s}+\alpha_{x} W_{x}^{T} W_{x}+\alpha_{z} W_{z}^{T} W_{z}
$$

Each component matrix in equation (3) can be written as the product of three individual matrices and one coefficient, and the detailed composition of them are mentioned in Li and Oldenburg [5].

The reference model ${ }^{m_{r e f}}$ may be a general background model that is estimated from previous investigations, or it could be the constant model. Here, the depth-weighting function is also incorporated to eliminate the skin effect which often occurs in the inversion of potential field data.

There are various kinds of constraints that can be incorporated here, and the fundamental constraint that used is the data misfit function which means that the data forwarded by the inverted model can fit the observed data in a settled precision, and it is wrote as

$$
\phi_{d}=\left\|W_{d}\left(G m-d_{o b s}\right)\right\|^{2}
$$

Where, matrix $G$ is the kernel function in equation (1), $d_{o b s}$ is the observed data set and $W_{d}$ is a diagonal matrix whose ith element is the reciprocal of the standard deviation of the ith datum. Meanwhile, some other constraints are also used to get more enhanced results. Here, researchers adopt bounds constraint and some conventional regularization terms. These regularization technique not only controls those parts of the inversion results that are not well constrained by the data, but also provides stability in the computational implementation.

\section{Data Experiments}

To verify validity of the algorithm, researchers implemented the developed algorithm to the inversion of synthetic gravity data. The data was forwarded from the theoretical models (Fig. 1). 
(a)

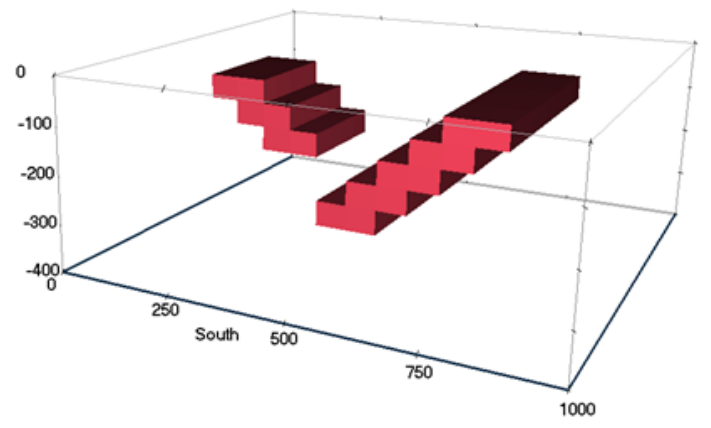

(b)

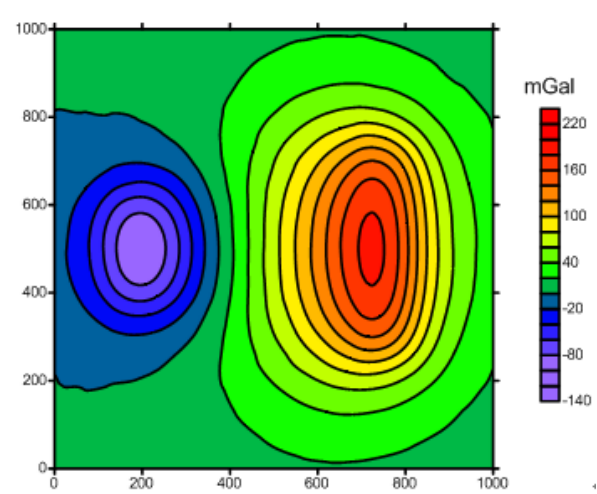

Fig. 1 (a) Perspective view of the thereotical model; (b) Gravty anomaly produced by the model.
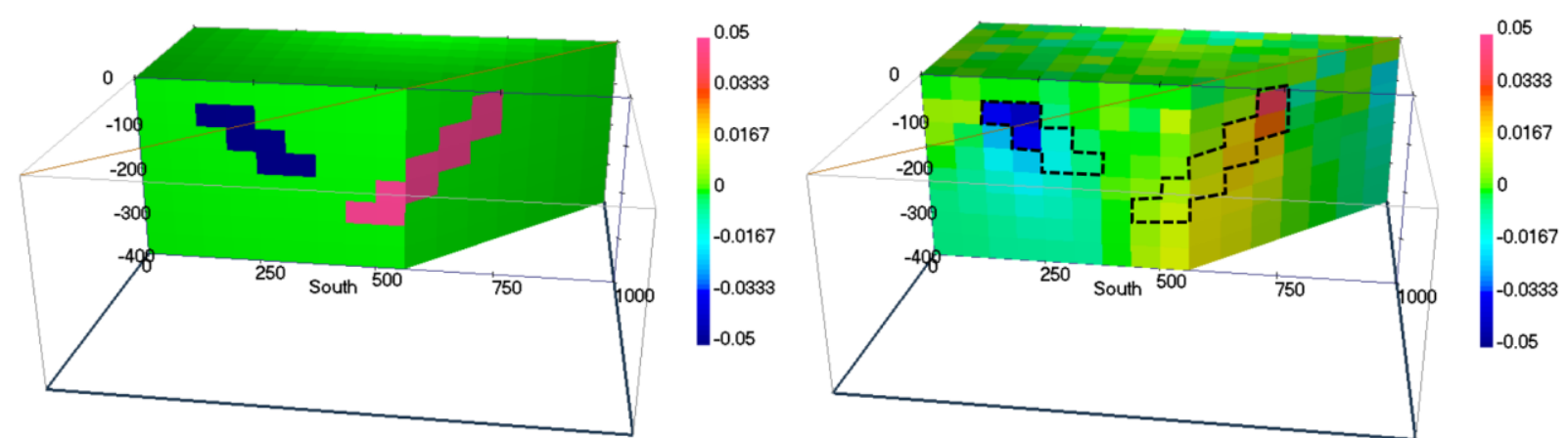

Fig. 2 The left figure is the slice of the theoretical model and the right figure is the same slice of the inverted result.

Then researchers applied the introduced algorithm to the synthetic gravity data. Fig. 2 shows the inverted model of this test. The same slice of the inverted model shows that it is very similar to the synthetic model, which proves the effectiveness of the introduced algorithm.

\section{Summary}

Researchers introduced the algorithm of 3D inversion of potential field data. And a synthetic model was utilized to test the effectiveness of this algorithm. The introduced algorithm can well shape the subsurface and it can be applied to the practical engineering.

\section{Acknowledgement}

This work was supported by 1) The National Natural Science Foundation of China, 41474106, 41374093 and 41074095 ; 2) The Major National scientific research and equipment development project, ZDYZ2012-1-02-04; 3) The Specialized Research Fund for the Doctoral Program of Higher Education of China, 20130022110011; 4) R\&D of Key Instruments and Technologies for Deep Resources Prospecting (the National R\&D Projects for Key Scientific Instruments), ZDYZ2012-1-02-04.

\section{References}

[1] M. Commer, G. A. Newman, New Advance in Three-dimensional Controlled-source Electromagnetic Inversion. Geophysical Journal International, 2008, 172(2): 513-535.

[2] L. H. Guo, X. H. Meng, Shi L. et al., 3D Correlation Imaging for Gravity and Gravity Gradiometry Data. Chinese Journal of Geophysics (in Chinese), 2009, 52(4), 1098-1106. 
[3] X. H. Meng, G. F. Liu G, Z. X. Chen, et al., 3-D Dravity and Magnetic Inversion for Physical Properties based on Residual Anomaly Correlation. Chinese Journal of Geophysics (in Chinese), 2012, 55(1): 304-309.

[4] Y. G. Li, D. W. Oldenburg, 3-D Inversion of Gravity Data. Geophysics. 1998, 63(1), 109-119.

[5] Y. G. Li, D. W. Oldenburg, 3-D Inversion of Magnetic Data. Geophysics. 1996, 61(2), 394-408.

[6] Y. G. Li , D. W. Oldenburg, Fast Inversion of Large-scale Magnetic Data Using Wavelet Transform and a Logarithmic Barrier Method. Geophys. J. Int. 2003, 152, 251-265.

[7] M. Moorkamp, M. Jegen, A. Robert, Massively Parallel Forward Modeling of Scalar and Tensor Gravimetry Data. Computers \& Geosciences, 2010, 36 (5). pp. 680-686.

[8] V. Palletti, S. Ialongo, G. Florio, et al., Self-constrained Inversion of Potential Fields. Geophysical Journal International, 2013, 195, 854-869. 